\title{
Design of a new planetary gear powertrain for vessel combined power
}

\author{
Fuchun Yang ${ }^{1,2, a}$, Fuxin Du ${ }^{1, b}$ \\ ${ }^{1}$ Department of Mechanical Engineering, Shandong University, Jinan, 250061, China \\ ${ }^{2}$ Key Laboratory of High-efficiency and Clean Mechanical Manufacture at Shandong University, \\ Ministry of Education, Jinan, 250061, China \\ aemail: yfc26@163.com, bemail:dufuxin@sdu.edu.cn
}

Keywords: Vessel; combined power; planetary gear; power flow

\begin{abstract}
With the development of modern vessels, higher requirements are put forward, such as higher power density, power split and power confluence properties. A new planetary gear powertrain for power combined vessels was proposed. The system has characteristics of multi-flow transmission, multi-ratio, multi-redundency and high power density. The power flow analysis based on hypergraph was studied. The status and power flow of the new system were analyzed based on the proposed method.
\end{abstract}

\section{Introduction}

Vessel combined powertrain is a propulsion system by changing the prime motors, propeller and operating modes according to the running status of the vessels. Gear powertrain is the key component in the combined powertrain system which can be controlled to satisfy different reduction and combining requirements. The current gear powertrains are mainly composed of fixed-axis gears, which have disadvantages such as lower power density, large volume and large weight. Planetary gear sets have great advantages in the control of power density, volume, mass and the power split and confluence. Therefore, a new planetary gear train will be proposed in this paper to satisfy the new need of vessel combined power.

According to types of prime motors, the current power combined systems are mainly composed of five types, that is, diesel and diesel (CODAD), diesel and gas turbine (CODAG), diesel or gas turbine (CODOG), diesel electricity and (or) gas turbine (CODELAG/CODELOG), diesel electricity and gas turbine electricity (CODEG) [1] [2]. The power split and power confluence in all these systems are performed by fixed-axis gear trains. Hoppe [3] pointed out that the development of vessels need gear trains with the properties of multi-input multi-output, compact, higher power density to satisfy the requirements of higher speed and less space.

The design work becomes very complex and time-consuming for complex planetary gear trains with more power flow changes and more reduction ratios. The researchers proposed different method to solve this problem. Rao [4] analyzed the planetary gear train by graphical method using branch nodes; Esmail et al [5] studies the kinematics and power flow of hybrid transmission and multi-input multi-output planetary gear trains by structure diagram; Goma-Ayats et al [6] studied the kinematics of gear trains with planetary gear by hypergraphs, the method is very clear to represent the gear train, but the research was limited to the kinematics analysis of one degree of freedom systems.

As mentioned above, the current powertrains for vessel combined power are mainly composed of fixed-axis gear sets, therefore, it's urgent to develop new combined powertrain systems with planetary gear sets for modern vessels. A new combined powertrain with planetary gear sets was proposed in this paper and its power flow and operating modes were also studied.

\section{System design of vessel combined powertrain}

The proposed planetary gear train for vessel combined power is shown in Figure 1. The system is mainly composed of cross-planetary gear train and compound planetary gear train, which make the 
system easy to realize multi-input multi-output, compact structure, multi-gear ratios, power split and power confluence. The cross-planetary gear train is shown in Figure 1(a) which is composed by a carrier, a sun gear and the ring gear $\mathrm{C}$ which possesses inner gear teeth and outer gear teeth at the same time. Based on these two types of planetary gear train and modularization theory, a complex combined powertrain can be achieved as shown in Figure 1 (b) to improve the life, redundency and maneuverability of vessels. By adding the components in dashed lines in Figure 1 (b), the system can be expanded easily to satisfy different propulsion demands.

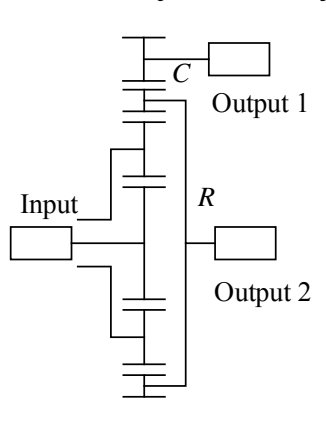

(a)

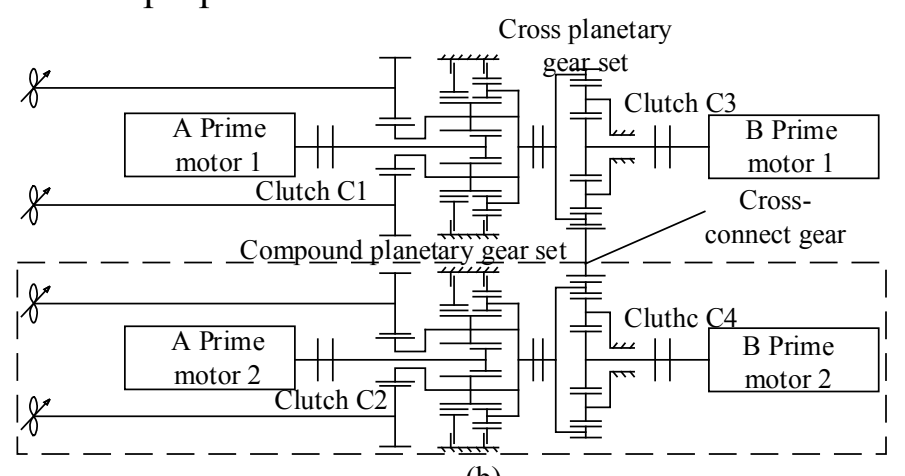

(b)

Fig.1 Powertrain (a) cross planetary gear set; (b) combined powertrain

\section{Power flow analysis based on hypergraph}

Planetary gear trains generally consist of three central shafts (one carrier, two central gears) and one group or several groups of planet gears. A planetary gear set is shown in Figure 2 (a) and its hypergraph is shown in Figure 2 (b). In Figure 2 (b), the round node represents a shaft in the mechanism, and the line represents the mechanical connection between two shafts. Its hypergraph is named by a letter, numerals 1 (for sun gear) and 3 (for ring gear) following its name represent the gear shafts; for example, $\mathrm{x} 1$ represents gear 1 . Numeral 2 following its name represents the carrier, for example, $\mathrm{x} 2$ represents the carrier 2 in planetary gear set $\mathrm{x}$. The characteristic gear ratio is represented by $\mathrm{k}$ with its name as subscript and it is the ratio between gearing shaft 1 and 3 when carrier shaft 2 is fixed, as shown in Figure 2 (b). The direction of the transmission is represented by an arrow.

A fixed-axis gear set as shown in Figure 2 (c) generally consists of two gears and its hypergraph is shown in Figure 2 (d). It's hypergraph is named by a letter and two gears are represented by numerals 1 and 3 following its name. The characteristic gear ratio is represented by $\mathrm{k}$ with its name as subscript and it is the ratio between gearing shaft 1 and 3. The direction of the transmission is represented by an arrow.

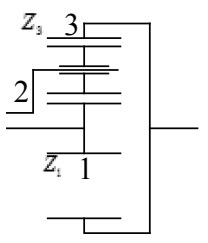

(a)

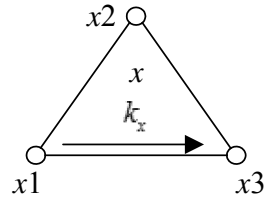

(b)

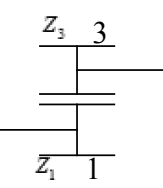

(c)

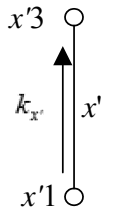

(d)

Fig.2 Gear sets and hypergraphs (a) planetary gear set; (b) hypergraph of (a); (c) fixed-axis gear set; (d) hypergraph of (c)

Based on the above definitions, the hypergraph of vessel combined powertrain in shown in Figure 3, where a1, e1 represent input power, m3, n3 represent output power, the filled circle represent a fixed component.

The kinematic relationship of a simple planetary gear set and a fixed-axis gear set can be expressed as following respectively,

$$
\begin{aligned}
& \omega_{x 1}=k_{x} \omega_{x 3}+\left(1-k_{x}\right) \omega_{x 2} \\
& \omega_{x 1}=k_{x} \omega_{x 3}
\end{aligned}
$$

In addition, for a complex hypergraph there are other kinematic relationships between connected components, such as $\omega_{b 3}=\omega_{c 3}, \omega_{a 2}=0$ in Figure 3 . Therefore, all the kinematic relationships of a 
hypergraph can be rewritten as matrix form, that is,

\section{$K \boldsymbol{\omega}=\boldsymbol{F}$}

Where $\boldsymbol{K}$ is a constant matrix with gear ratios, $\boldsymbol{F}$ is the vector with input velocity, $\boldsymbol{\omega}$ is the velocity vector, then, the velocities of each components can be obtained as

$\omega=K^{-1} \boldsymbol{F}$

Then, the ratio between any two components can be obtained by Equation (3). The kinematics of any other types of gear trains can be analyzed by the above method.

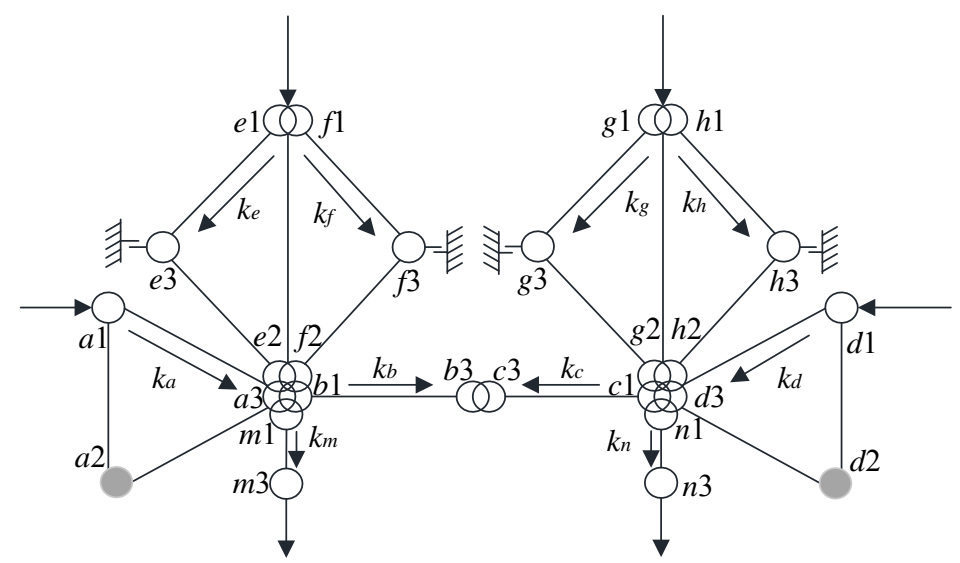

Fig.3 Hypergraph of Figure 1

Ignoring the power loss of planetary gear set and fixed-axis gear set, their torque and power relationships are obtained as following respectively,

$$
\begin{aligned}
& \left\{\begin{array}{l}
T_{x 1}+T_{x 2}+T_{x 3}=0 \\
T_{x 1} \omega_{x 1}+T_{x 2} \omega_{x 2}+T_{x 3} \omega_{x 3}=0
\end{array}\right. \\
& T_{x 1} \omega_{x 1}+T_{x 3} \omega_{x 3}=0
\end{aligned}
$$

Based on the connection relations, for instance, the total torque should be zero, multi-input components have input power ratio, the other equations can be obtained, such as $T_{a 3}+T_{e 2}+T_{m 1}+T_{b 1}=0$ in Figure 3. Therefore, the torque and power equations can be written in matrix forms, that is,

$$
\boldsymbol{I T}=\boldsymbol{D}
$$

Where $\boldsymbol{I}$ is the constant matrix with velocities, $\boldsymbol{T}$ is torque vector, $\boldsymbol{D}$ is the constant vector with load torques, then the torques of each component can be get as

$\boldsymbol{T}=\boldsymbol{I}^{-1} \boldsymbol{D}$

The power of each component can be obtained by the computation in Equation (3) and Equation (6), that is

\section{$P=\omega T$}

The direction of power flow can be determined by the rules: 1) $P_{i j}>0$, power flows in the node; 2) $P_{i j}<0$, power flows out the node; 3 ) $P_{i j}=0$, the power passes through the node. Therefore, the power flow of the whole system can be obtained by the above computation and rules.

\section{System operation modes and power flow}

The operation types of vessel combined powertrain in Figure 1 (b) mainly include six types, that is single prime motor operating mode, double prime motors operating mode, three prime motors operating mode and all prime motors operating mode, as shown in Table 1, where the $\mathrm{X}$ in bracket means or.

Table 1 Operating modes of the system

\begin{tabular}{ccccccc}
\hline Prime motor & Mode 1 & Mode 2 & Mode 3 & Mode 4 & Mode 5 & Mode 6 \\
\hline A1 & $\mathrm{X}$ & & $\mathrm{X}$ & $\mathrm{X}$ & $\mathrm{X}$ & $\mathrm{X}$ \\
A2 & $(\mathrm{X})$ & & $(\mathrm{X})$ & $\mathrm{X}$ & $(\mathrm{X})$ & $\mathrm{X}$ \\
$\mathrm{B} 1$ & & $\mathrm{X}$ & $\mathrm{X}$ & $\mathrm{X}$ & $\mathrm{X}$ & $\mathrm{X}$ \\
$\mathrm{B} 2$ & & $(\mathrm{X})$ & $(\mathrm{X})$ & $(\mathrm{X})$ & $\mathrm{X}$ & $\mathrm{X}$ \\
\hline
\end{tabular}


This paper will discuss the power flow of mode 3 and mode 6 by the method proposed in Section 3. The gear ratio of each basic gear set is shown in Table 2 and the input velocities and load torques are all processed by dimensionless method.

Table 2 Ratio of each basic gear set

\begin{tabular}{ccccc}
\hline$k_{a} / k_{d}$ & $k_{b} / k_{c}$ & $k_{e} / k_{q}$ & $k_{f} / k_{h}$ & $k_{m} / k_{n}$ \\
\hline-2.2 & -1 & -1.8 & -2.4 & -1.6 \\
\hline
\end{tabular}

In operating mode 3 , the input is single motor and the output is double loads. The output load torques are assumed the same. Therefore, the kinematics, torque and power relations of operating mode 3( $a 1$ input, $m 3, n 3$ output) can be obtained as

$$
\left\{\begin{array} { l l } 
{ \omega _ { a 1 } = k _ { a } \omega _ { a 3 } + ( 1 - k _ { a } ) \omega _ { a 2 } } \\
{ \omega _ { m 1 } = k _ { m } \omega _ { m 3 } } \\
{ \omega _ { n 1 } = k _ { n } \omega _ { n 3 } } \\
{ \omega _ { b 1 } = k _ { b } \omega _ { b 3 } } \\
{ \omega _ { c 1 } = k _ { c } \omega _ { c 3 } } \\
{ \omega _ { a 3 } = \omega _ { b 1 } } \\
{ \omega _ { a 3 } = \omega _ { m 1 } } \\
{ \omega _ { b 2 } = \omega _ { c 2 } } \\
{ \omega _ { c 1 } = \omega _ { g 2 } } \\
{ \omega _ { a 2 } = 0 } \\
{ \omega _ { a 1 } = \omega _ { 0 } }
\end{array} \quad \left\{\begin{array}{l}
T_{a 1}+T_{a 2}+T_{a 3}=0 \\
T_{a 1} \omega_{a 1}+T_{a 2} \omega_{a 2}+T_{a 3} \omega_{a 3}=0 \\
T_{m 1} \omega_{m 1}+T_{m 3} \omega_{m 3}=0 \\
T_{n 1} \omega_{n 1}+T_{n 3} \omega_{n 3}=0 \\
T_{b 1} \omega_{b 1}+T_{b 3} \omega_{b 3}=0 \\
T_{c 1} \omega_{c 1}+T_{c 3} \omega_{c 3}=0 \\
T_{a 3}+T_{m 1}+T_{b 1}=0 \\
T_{n 1}+T_{c 1}=0 \\
T_{b 2}+T_{c 2}=0 \\
T_{m 3}=T_{0} \\
T_{n 3}=T_{0}
\end{array}\right.\right.
$$

The power flow of each components in operating mode 3 are shown in Figure 4 by solving the above equations in matrix form. In Figure 4, it can be observed that the input power is split to output components $m$ and $n$ by cross-gears $b$ and $c$ to realize the power split in operating mode 3 . The split power on each output component is determined by its load torque.

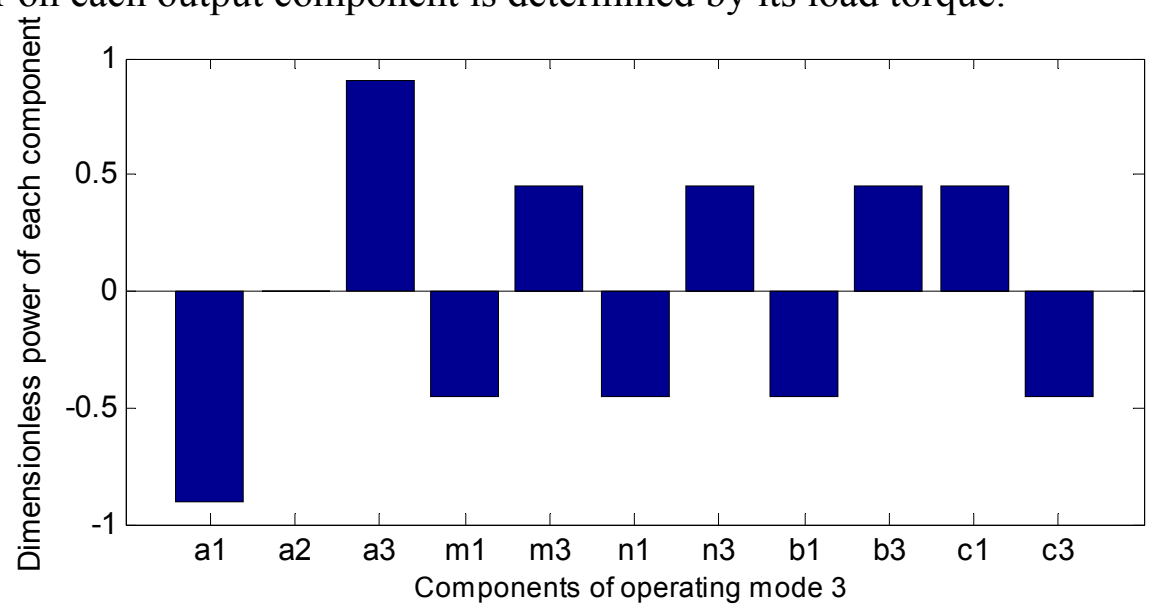

Fig. 4 Power flow of operating mode 3

Operating mode 6 is an operating mode with multi-input and multi-output, the input powers distribution on input components $a 1, d 1, e 1$, and $g 1$ are determined by power distribution coefficients $\gamma_{1}$ and $\gamma_{2}$, here it is given as $\gamma_{1}=0.6, \gamma_{2}=1$ and the load torques are assumed the same. Therefore, the kinematic and power can be obtained by the method proposed above (where $a 1, e 1$, $d 1, g 1$ are input components, $m 3, n 3$ are output components) as 


$$
\begin{cases}\omega_{a 1}=k_{a} \omega_{a 3}+\left(1-k_{a}\right) \omega_{a 2} \\ \omega_{d 1}=k_{d} \omega_{d 3}+\left(1-k_{d}\right) \omega_{d 2} \\ \omega_{e 1}=k_{e} \omega_{e 3}+\left(1-k_{e}\right) \omega_{e 2} \\ \omega_{g 1}=k_{g} \omega_{g 3}+\left(1-k_{g}\right) \omega_{g 2} \\ \omega_{m 1}=k_{m} \omega_{m 3} \\ \omega_{n 1}=k_{n} \omega_{n 3} \\ \omega_{b 1}=k_{b} \omega_{b 3} \\ \omega_{c 1}=k_{c} \omega_{c 3} \\ \omega_{a 3}=\omega_{e 2} \\ \omega_{a 3}=\omega_{b 1} \\ \omega_{a 3}=\omega_{m 1} \\ \omega_{b 2}=\omega_{c 2} \\ \omega_{d 3}=\omega_{g 2} \\ \omega_{d 3}=\omega_{c 1} \\ \omega_{d 3}=\omega_{n 1} \\ \omega_{a 2}=0 \\ \omega_{e 3}=0 \\ \omega_{d 2}=0 \\ \omega_{g 3}=0 \\ \omega_{a 1}=\omega_{d 1}+T_{d 2}+T_{d 3}=0 \\ T_{d 1} \omega_{d 1}+T_{d 2} \omega_{d 2}+T_{d 3} \omega_{d 3}=0 \\ T_{e 1}+T_{e 2}+T_{e 3}=0 \\ T_{e 1} \omega_{e 1}+T_{e 2} \omega_{e 2}+T_{e 3} \omega_{e 3}=0 \\ T_{g 1}+T_{g 2}+T_{g 3}=0 \\ T_{g 1} \omega_{g 1}+T_{g 2} \omega_{g 2}+T_{g 3} \omega_{g 3}=0 \\ T_{m 1} \omega_{m 1}+T_{m 3} \omega_{m 3}=0 \\ T_{n 1} \omega_{n 1}+T_{n 3} \omega_{n 3}=0 \\ T_{b 1} \omega_{b 1}+T_{b 3} \omega_{b 3}=0 \\ T_{c 1} \omega_{c 1}+T_{c 3} \omega_{c 3}=0 \\ T_{a 3}+T_{e 2}+T_{m 1}+T_{b 1}=0 \\ T_{d 3}+T_{g 2}+T_{n 1}+T_{c 1}=0 \\ T_{b 2}+T_{c 2}=0 \\ T_{a 1} \omega_{a 1}=\gamma_{1} T_{e 1} \omega_{e 1} \\ T_{d 1} \omega_{d 1}=\gamma_{1} T_{g 1} \omega_{g 1} \\ T_{e 1} \omega_{e 1}=\gamma_{2} T_{g 1} \omega_{g 1} \\ T_{m 3}=T_{0} \\ T_{n 3}=T_{0} \\ T_{a}\end{cases}
$$

The power flow of each components in operating mode 6 are shown in Figure 5 by solving the above equations in matrix form. In Figure 5 , it can be observed that the four inputs are transferred to two output components $m 3$ and $n 3$, in which the cross gears $b$ and $c$ don't participate in the power distribution and their powers are all zero.

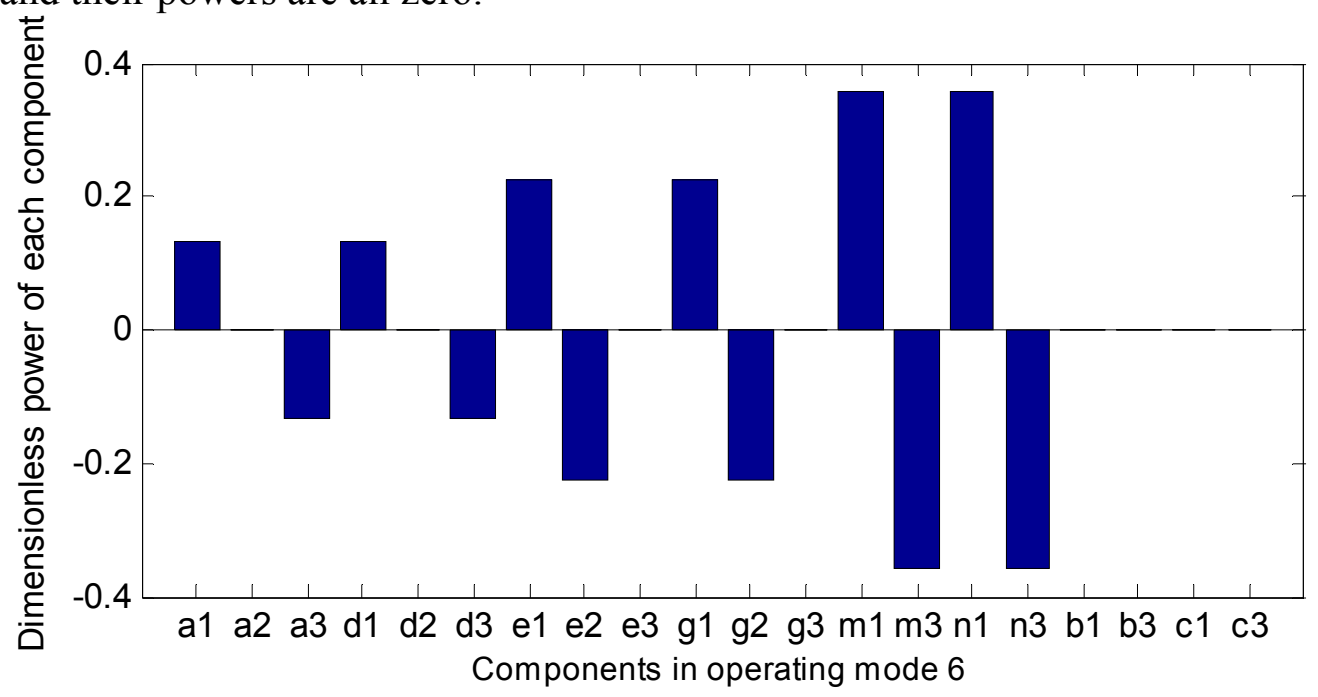

Fig.5. Power flow of operating mode 6

\section{Conclusion}

A new vessel combined powertrain with planetary gear sets was proposed in this paper, this system has properties of multi-flow transmission, multi-reduction ratio, multi-redundency and high power density. Its power flow was analyzed by hypergraph method and the equation establishment 
was also studied. The operating mode of the system were analyzed and the power flow of two typical operating mode were studied. The new system provides a new design idea in combined powertrain for different vessels.

\section{Acknowledgement}

This paper was supported by National Natural Science Foundation of China (Grant No.51305233), Research Fund for the Doctoral Program of Higher Education of China (Grant No.20120131120056).

\section{References}

[1] Status and future of marine propulsion solution. Dip. Ing. Hubert. Ohmayer, 2008.

[2] Renk gear sets for latest German frigates. Marine propulsion \& auxiliary machinery, 2009.

[3] Hoppe, F. Naval gear systems and their future demands. Conference Proceedings of the Institute of Marine Engineering, Science and Technology - INEC 2008: Embracing the Future.

[4] Rao, Y., Rao, A. Generation of epicyclic gear trains of one degree of freedom. ASME Journal of mechanical design, 2008, 130(5): 052604-8.

[5] Esmail, E. An approach to power-flow and static force analysis in multi-input multi-output epicyclic-type transmission trains. Journal of mechanical design, 2010, 132: 011009-10.

[6] Goma-Ayats, J.R., Diego-Ayala, U., Canela, et al. Hypergraphs for the analysis of complex mechanisms comprising planetary gear trains and other variable or fixed transmissions. Mechanism and machine theory, 2012, 51:217-229. 